\title{
Conceitos de enfermeiros e médicos de um serviço de emergência sobre dor e analgesia no trauma*
}

\author{
CONCEPTS OF EMERGENCY SERVICE NURSES AND PHYSICIANS \\ REGARDING PAIN AND ANALGESIA IN TRAUMA
}

\author{
CONCEPTOS DE ENFERMEROS Y MÉDICOS DE UN SERVICIO DE EMERGENCIA \\ SOBRE DOLOR Y ANALGESIA EN EL TRAUMA
}

\section{Ana Maria Calil', Cibele Andrucioli de Mattos Pimenta ${ }^{2}$}

\section{RESUMO}

Analisaram-se os conceitos sobre dor e analgesia no trauma de enfermeiros e médicos de um serviço de emergência. Foram realizadas entrevistas semiestruturadas com $100 \%$ da equipe de enfermeiros (22) e $85 \%$ da equipe médica (22) do ProntoSocorro Cirúrgico do HCFMUSP. A análise dos dados apontou alguns conceitos concordantes e outros discor-dantes com a literatura. Os profissionais estavam de acordo na maior parte dos conceitos, mas diferiram em alguns. Na opinião dos profissionais, baixa prioridade é dada ao controle da dor no trauma.

\section{DESCRITORES}

Dor.

Analgesia.

Ferimentos e lesões (enfermagem). Emergências.

\begin{abstract}
This study analyzed the concepts of pain and analgesia in trauma nurses and physicians of an emergency service have. Semistructured interviews were carried out with 100 percent of the nursing staff (22) and 85 percent of the medical staff (22) of the Surgical First Aid Service of the University of São Paulo Medical School's Hospital das Clínicas. The analysis of the data revealed that some of the ideas coincide with the literature, but others do not. The interviewees agreed with most of the concepts, but disagreed with some. In the professionals' opinion, little priority is given to the control of pain in trauma.
\end{abstract}

\section{KEY WORDS}

Pain.

Analgesia.

Wounds and injuries (nursing).

Emergency.

\section{RESUMEN}

En esta investigación se analizaron los conceptos que sobre dolor y analgesia, en el trauma, tienen enfermeros y médicos de un servicio de emergencia. Fueron realizadas entrevistas semiestructuradas con el $100 \%$ del equipo de enfermeros (22) y el $85 \%$ del equipo médico (22) del Servicio de Emergencias Quirúrgicas del HCFMUSP. El análisis de los datos apuntó algunos conceptos concordantes y otros discordantes con la literatura. Los profesionales estaban de acuerdo con la mayor parte de los conceptos, sin embargo diferían en algunos. En la opinión de los profesionales, se da una baja prioridad al control del dolor en el trauma.

\section{DESCRIPTORES}

Dolor.

Analgesia.

Heridas y traumatismos

(enfermería).

Urgencias médicas
* Extraído da Tese de Doutorado "Dor e analgesia em vítimas de acidente de transporte atendidas em um pronto-socorro". São Paulo, 2003.

1 Doutora em Enfermagem pela Escola de Enfermagem da Universidade de São Paulo (EEUSP). easallum.fnr@terra.com.br

${ }^{2}$ Enfermeira. Prof ${ }^{a}$ LivreDocente do Depto de Enfermagem MédicoCirúrgica da EEUSP. parpca@usp.br 
Ana Maria Calil

Cibele A. de M. Pimenta

\section{INTRODUÇÃO}

Dor é uma das principais conseqüências do trauma e suas repercussões são potencialmente prejudiciais ao organismo ${ }^{(1-2)}$. Embora freqüente, pouca atenção é dada ao controle álgico do traumatizado e o tema é insuficientemente conhecido $^{(3)}$.

As razões para o inadequado controle da dor no trauma são diversas. As repercussões orgânicas do processo álgico intenso são subestimadas por médicos e enfermeiros; há grande desinformação sobre as técnicas disponíveis e sobre a farmacologia das drogas analgésicas; alega-se que as condições do local do acidente e do setor de emergência são adversas a esse tipo de tratamento e que a administração precoce de analgésicos pode mascarar indício valioso para o diagnóstico etiológico ${ }^{(2)}$.

Dor é uma experiência sensorial e emocional desagradável, associada a uma lesão tissular real ou potencial, e descrita em termos de tal dano ${ }^{(4)}$. A dor aguda está relacionada a afecções mecânicas, químicas e térmicas, como aquelas advindas de traumas, queimaduras, infecções e processos inflamatórios $^{(1,5)}$.

A evolução natural da dor aguda é a remissão. É mais intensa no início e, à medida que ocorre a restauração tecidual, há redução da síntese de substâncias algiogênicas e diminuição da dor ${ }^{(5)}$. Há especulações de que, decorrente da ativação prolongada de várias vias neuronais, a dor aguda possa se cronificar $^{(6)}$.

A dor aguda não aliviada pode ocasionar: a elevação da pressão arterial, o aumento da freqüência cardíaca e respiratória, entre outras, que podem resultar em hipoventilação, aumento do trabalho cardíaco e diminuição da perfusão sangüínea periférica. Nos quadros hemorrágicos os estímulos nociceptivos podem agravar o choque, pelo aumento do trabalho do ventrículo esquerdo e redução da oferta de oxigênio ${ }^{(7)}$. O controle da dor, além de humanitário, é vital para a assistência imediata ao acidentado, visando manter as funções fisiológicas básicas e evitar efeitos deletérios.

O tratamento da dor no setor de emergência é complexo, e isso se deve a fatores como a subjetividade do fenômeno, as diferenças quanto ao sexo e raça, local, tipo e gravidade da lesão, intensidade e local de dor, e tipo e quantidade de medicamentos, entre outros. No entanto, nenhum desses fatores justifica ações negligentes ${ }^{(8)}$.
No setor de emergência grande parte dos atendimentos é decorrente de causas externas, constituídas por acidentes e violências, conforme Classificação Estatística Internacional de Doenças e Problemas Relacionados à Saúde (CID-10) ${ }^{(9)}$. Causas Externas são um problema de Saúde Pública em todo o mundo, pois são responsáveis por parte substancial da morbidade, mortalidade e incapacidades, além do considerável custo sócio-econômico ${ }^{(10-11)}$. No Brasil, constituem-se na segunda causa de mortalidade geral da população, desconsideradas as causas mal definidas $^{(12-13)}$.

Entre as dores agudas, a dor na emergência é a menos investigada em nosso meio. Tal fato é preocupante, visto o grande número de vítimas que são atendidas e permanecem diariamente nesse setor. Além disso, não existe, nos manuais de atendimento às vítimas de trauma, um capítulo dedicado ao tema dor.

Em um estudo ${ }^{(14)}$ analisou-se a prevalência e as características da dor em 139 vítimas de acidentes de causa externa na fase pré-hospitalar; descreveu-se os recursos humanos utilizados no socorro, os medicamentos analgésicos existentes nas viaturas e os procedimentos de analgesia implementados. Este estudo foi feito no prontosocorro do Hospital das Clínicas da FMUSP durante 12 finais de semana. Todas as vítimas de acidentes de causa externa removidas por viaturas de saúde, admitidas na sala de urgência do pronto-socorro e a equipe envolvida no resgate foram entrevistadas. Os traumatizados foram homens $(77,7 \%)$ e a faixa etária predominante foi a de menor ou igual a 40 anos $(69,0 \%)$. Havia queixa de dor no momento do trauma em $67(48,2 \%)$ das vítimas e, no momento da entrevista, em 87 $(62,6 \%)$ delas. Analgesia episódica ocorreu em $13,9 \%$ dos entrevistados. A dor foi avaliada como intensa por $44 \%$ das vítimas, como moderada por $21,5 \%$ e era insuportável em $19 \%$ das vezes. A média de intensidade de dor foi 6,5 . Os locais mais freqüentes de queixa álgica foram MMII $(30,6 \%)$, MMSS $(21,9 \%)$ e crânio $(18,1 \%)$, o que correspondeu ao local da lesão. A maioria dos profissionais $(75,5 \%)$ envolvidos na remoção foi bombeiros. Havia médico em $15 \%$ dos veículos e algum profissional de enfermagem em 21,6\%. A intervenção analgésica mais freqüente foi a imobilização (50,4\%). Em cerca de 14,5\% das viaturas havia medicação analgésica, que foi utilizada apenas em 4,3\% (6) das remoções.

Estudiosos em dor e trauma concordam que a avaliação e o tratamento dos mesmos é atribuição da equipe de saúde ${ }^{(15)}$. Desse modo, concei- 
tos fundamentais a respeito da dor e suas repercussões, das possibilidades diagnósticas e sobre os princípios de terapêutica devem ser conhecidos por aqueles que atuam em emergência.

Com a finalidade de avaliar o conhecimento sobre dor aguda e seu controle entre médicos e acadêmicos do $6^{\circ}$ ano no setor de emergência, estudiosos ${ }^{(16)}$ constataram que dos entrevistados, $50 \%$ consideraram-se em condições de tratar a dor na maioria das ocasiões, porém, expressiva proporção de internos e residentes $(40,6 \%)$ não se considerou em condições de fazê-lo. Além disso, proporção significativa dos entrevistados $(52,0 \%)$, disse prescrever analgésicos apenas quando necessário e não de forma regular. Esse fato é comum e preocupante em qualquer setor hospitalar, uma vez que, para o adequado alívio e tratamento da dor, são necessárias avaliações freqüentes, realizadas por profissional capacitado e documentadas em prontuário, por parte de toda equipe multiprofissional.

$\mathrm{O}$ estudo que analisou a dor no trauma na fase pré-hospitalar ${ }^{(14)}$ e o que analisou o conhecimento de médicos e estudantes de medicina sobre controle da dor no pronto-socorro ${ }^{(16)}$, mostram que também há relato de inadequado tratamento da dor na emergência em nosso meio. No presente estudo, optou-se pelos questionamentos: quais os critérios utilizados por enfermeiros e médicos para a indicação de terapêutica analgésica no trauma e qual é a ênfase conferida à analgesia no trauma.

\section{CASUÍSTICA E MÉTODO}

Trata-se de um estudo exploratório, que foi desenvolvido no Pronto Socorro Cirúrgico (PSC) do Hospital das Clínicas da Faculdade de Medicina da Universidade de São Paulo. O PSC é o primeiro local para $\mathrm{o}$ atendimento a pacientes de causas externas.

Após autorização da Comissão de Ética em Pesquisa da instituição, os dados foram coletados em maio de 2003. Todos os profissionais que concordaram em participar assinaram Termo de Consentimento Pós Informado em duas vias.

Todos os médicos e enfermeiros do serviço foram convidados a participar, no máximo, três vezes. A equipe médica foi composta por residentes do $3^{\circ}$ ano de cirurgia geral, assistentes e chefes de plantão, e foram incluídos os enfermeiros assistenciais e com cargo de chefia. Atenderam à solicitação 44 profissionais, 22 médicos e 22 enfermeiros, o que correspondeu a $85 \%$ dos médicos e $100 \%$ dos enfermeiros do PSC.
As entrevistas foram estruturadas, individuais e continham cinco questões.

- Em quais situações há indicação de analgesia em vítimas de trauma?

- Quais situações contra indicam analgesia nesses pacientes?

- Quais os principais parâmetros para se avaliar a eficácia da analgesia?

- Que ênfase é dada para a analgesia em traumatologia, numa escala de 0 a10, sendo 0 a ênfase mínima e 10 a ênfase máxima?

- Na sua opinião qual deveria ser esta ênfase, numa escala de 0 a 10 ?

As respostas foram analisadas e, por similaridade, organizadas em categorias. Para as questões que permitiram múltiplas respostas, todas foram listadas. Os resultados estão apresentados em tabelas, em números absolutos e relativos. A comparação das respostas entre médicos e enfermeiros foi feita por meio do Teste Qui-Quadrado, Teste de Fischer e o Teste não paramétrico de MannWhitney. Para a análise estatística, foram contempladas as cinco respostas mais freqüentes de cada grupo profissional.

\section{RESULTADOS E DISCUSSÃO}

Os dados obtidos estão apresentados na ordem das perguntas propostas.

Observa-se na Tabela 1 que os cinco maiores percentuais foram identificados para "presença de dor" (36,4\%), "após avaliação primária $\rightarrow$ todas as situações" $(29,5 \%)$, "fratura simples de membros" (29,5\%), "fraturas expostas" $(25,0 \%)$ e "pacientes conscientes" $(11,4 \%)$, com diferenças importantes entre os profissionais.

Observa-se, na Tabela 2, haver diferença significativa $(p<0,03)$ entre os profissionais em relação às respostas "presença de dor" e "após avaliação primária $\rightarrow$ todas as situações". Os enfermeiros apresentaram maior percentual em "presença de dor" e os médicos em "após avaliação primária $\rightarrow$ todas as situações".

A opinião dos profissionais (Tabela 1) quanto às situações para indicação de analgesia em vítimas de trauma, em sua maior parte, é concordante com a literatura ${ }^{(2-3,15)}$. Em ambas as categorias profissionais há indicação de analgesia para lesões na região corpórea de membros superiores e inferiores, mais especificamente fraturas simples e fratu-
Conceitos de enfermeiros e médicos de um serviço de emergência sobre dor e analgesia no trauma 
Ana Maria Calil Cibele A. de M. Pimenta ras expostas. No entanto, a freqüência com que algumas situações com alto potencial para dor foram citadas foi muito baixa ou nula, como no caso de lesões torácicas, lesões pélvicas, queimados e ferimentos descolantes. Para os queimados, citados apenas duas vezes, uma em cada categoria profissional, o alívio da dor é prioridade da assistência ${ }^{(15)}$. Esses dados, possivelmente, indicam o pouco enfoque conferido à analgesia no setor de emergência, e a pequena observação dos profissionais para as situações em que a dor é um evento freqüente e esperado.

Tabela 1 - Distribuição da opinião dos profissionais sobre as indicações para o uso de analgesia em pacientes de trauma. (São Paulo, 2002)

\begin{tabular}{|c|c|c|c|c|c|c|}
\hline \multirow{2}{*}{ Situação } & \multicolumn{2}{|c|}{ Enfermeiros } & \multicolumn{2}{|c|}{ Médicos } & \multicolumn{2}{|c|}{$\begin{array}{l}\text { Todos } \\
\text { Profissionais }\end{array}$} \\
\hline & $\mathbf{n}=\mathbf{2 2}$ & $\%$ & $\mathrm{n}=22$ & $\%$ & $\mathrm{n}=44$ & $\%$ \\
\hline Presença de dor & 14 & 63,6 & 2 & 9,1 & 16 & 36,4 \\
\hline Após avaliação primária - Todas as situações & 3 & 13,6 & 10 & 45,5 & 13 & 29,5 \\
\hline Fratura simples MMII ou MMSS & 7 & 31,8 & 6 & 27,3 & 13 & 29,5 \\
\hline Fraturas expostas & 8 & 36,4 & 3 & 13,6 & 11 & 25,0 \\
\hline Pacientes conscientes & 1 & 4,5 & 4 & 18,2 & 5 & 11,4 \\
\hline Todas (independentemente de qualquer fator e tempo) & 3 & 13,6 & - & - & 3 & 6,8 \\
\hline Agitação & 3 & 13,6 & - & - & 3 & 6,8 \\
\hline Amputação traumática & 1 & 4,5 & 2 & 9,1 & 3 & 6,8 \\
\hline Após diagnóstico definitivo & - & - & 3 & 13,6 & 3 & 6,8 \\
\hline Lesões pélvicas & - & - & 3 & 13,6 & 3 & 6,8 \\
\hline Ferimento corto-contuso & 2 & 9,1 & - & - & 2 & 4,5 \\
\hline Queimados & 1 & 4,5 & 1 & 4,5 & 2 & 4,5 \\
\hline Lesão torácicas & - & - & 2 & 9,1 & 2 & 4,5 \\
\hline Paciente hemodinamicamente estável & - & - & 2 & 9,1 & 2 & 4,5 \\
\hline Escoriações & 1 & 4,5 & - & - & 1 & 2,3 \\
\hline Ausência lesões abdominais & - & - & 1 & 4,5 & 1 & 2,3 \\
\hline Ausência lesões neurológicas & - & - & 1 & 4,5 & 1 & 2,3 \\
\hline Ferimento descolante & - & - & 1 & 4,5 & 1 & 2,3 \\
\hline
\end{tabular}

Tabela 2 - Síntese das comparações das cinco respostas mais freqüentes apresentadas pelos profissionais na Tabela 1. (São Paulo, 2002)

\begin{tabular}{ll}
\hline Respostas mais freqüentes & P \\
\hline Presença de dor & 0,001 \\
Após avaliação primária - Todas as situações & 0,021 \\
Fratura simples MMII ou MMSS & 0,741 \\
Fraturas expostas & 0,082 \\
Pacientes conscientes & $0,345^{*}$ \\
\hline
\end{tabular}

* Teste exato de Fisher

A opinião dos profissionais quanto à indicação para analgesia diferiu significativamente (Tabela 1 e 2). Analgesiar quando o doente referir dor foi considerada pela maior parte dos enfermeiros $(63,6 \%)$, e por pequena parte dos médi$\cos (9,1 \%)$.

Algumas possibilidades para essas diferenças foram pensadas e um fator importante para tal distinção pode ter origem na formação acadêmica.

Historicamente, durante a formação do enfermeiro, é dada ênfase para a humanização e o processo de interação e inter-relação pessoais. Além disso, cabe ao profissional de enfermagem, na rotina hospitalar, maior proximidade com o paciente. Esses fatores parecem contribuir para que o enfermeiro "ouça" mais o paciente e atue de modo mais compartilhado ${ }^{(17-18)}$.

Outro aspecto interessante refere-se ao próprio conceito de dor que a define como um fenômeno subjetivo e, como tal, só pode ser indicado e quantificado pelo sujeito que a sente. Nesse sentido, os enfermeiros mostraram-se mais concordantes quanto ao conceito de dor que os médicos.

Verifica-se, na Tabela 3, que as cinco razões mais freqüentes para contra-indicar a analgesia foram: "nenhuma situação" $(25,0 \%)$, "escore na Escala de Coma de Glasgow < 9" $(25,0 \%)$, "trauma crânioencefálico" (20,5\%), "lesões abdominais" $(15,9 \%)$ e "analgesia mascarar o quadro" $(13,6 \%)$.
Rev Esc Enferm USP 2005; 39(3):325-32 
Tabela 3 - Distribuição da opinião dos profissionais sobre as contra-indicações para o uso de analgesia em pacientes de trauma. (São Paulo, 2002)

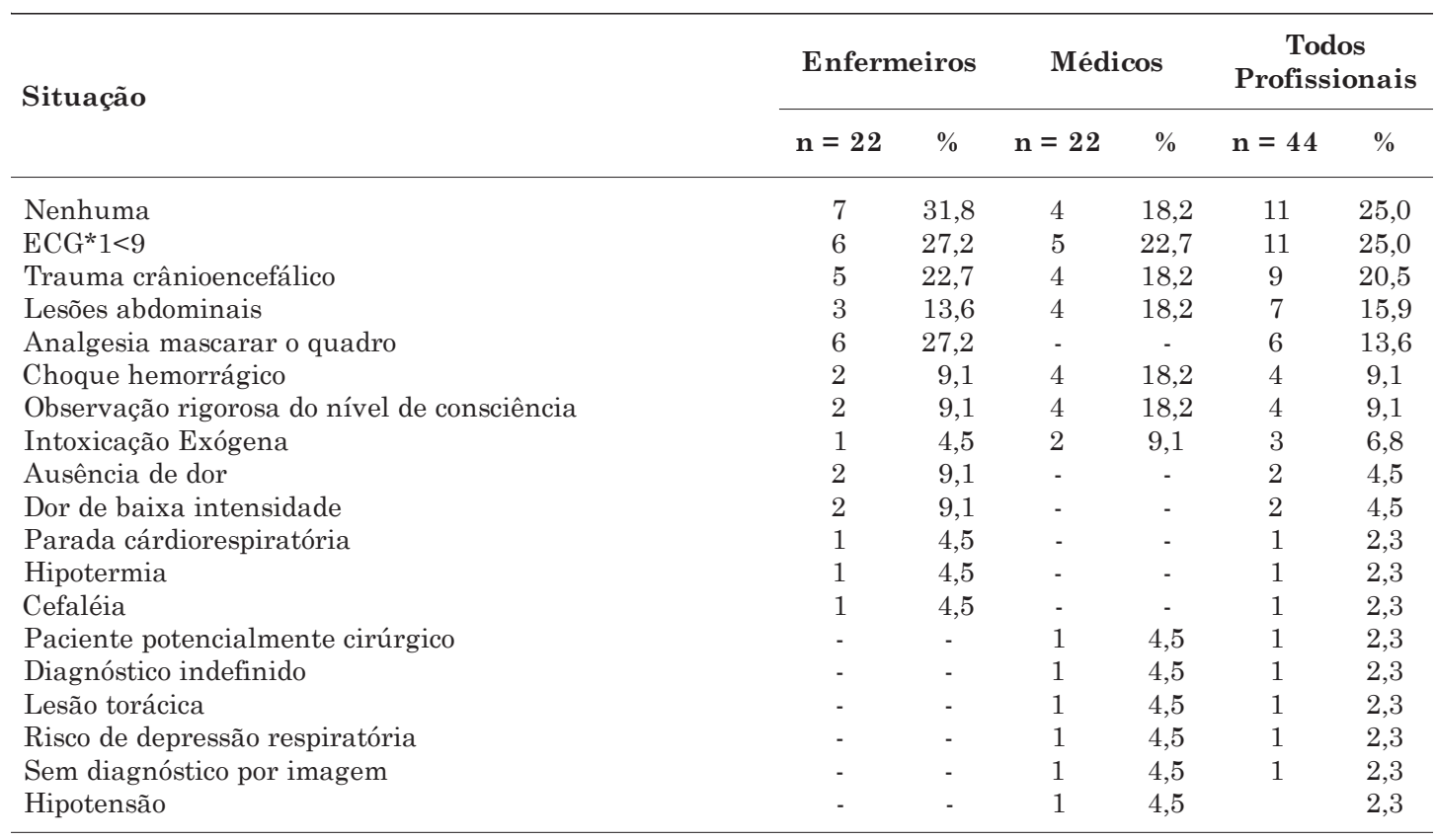

Conceitos de enfermeiros e médicos de um serviço de emergência sobre dor e analgesia no trauma

* ECGl= Escala de Coma de Glasgow

Analisando a opinião de médicos e enfermeiros em relação às situações de contra indicação de analgesia para vítimas de trauma, verificou-se que "nenhuma situação" apareceu para $25 \%$ do total de profissionais (Tabela 3). Situações como "trauma crânio-encefálico" ou "observação do ní- vel de consciência" foram citadas por ambos profissionais. Pela Tabela 4 observa-se haver diferença significativa $(p=0,028)$ entre as duas categorias profissionais em relação à resposta "analgesia mascarar o quadro". Seis enfermeiros e nenhum médico consideraram tal possibilidade.

Tabela 4 - Síntese das comparações das cinco respostas mais freqüentes apresentadas pelos profissionais na Tabela 3. (São Paulo, 2002)

\begin{tabular}{lc}
\hline Respostas mais freqüentes & $\mathbf{P}$ \\
\hline Nenhuma situação & $0,296^{*}$ \\
ECG1<9 & 0,727 \\
Trauma crânioencefálico & 1,000 \\
Lesões abdominais & 1,000 \\
Analgesia mascarar o quadro & 0,028 \\
\hline
\end{tabular}

* Teste Qui-quadrado

Inúmeras outras situações para contra-indicação de analgesia foram citadas, com frequências baixas, por ambas categorias profissionais. Algumas são concordantes com a literatura (choque hemorrágico, hipotensão, risco de depressão respiratória) ${ }^{(19-20)}$, outras discordantes (dor de baixa intensidade, paciente potencialmente cirúrgico, diagnóstico indefinido, lesão torácica, cefaléia, sem diagnóstico por imagem) $)^{(21)}$, e outras discutíveis, como trauma crânioencefálico e observação rigorosa do nível de consciência ${ }^{(22-23)}$.
Médicos e enfermeiros mostraram algumas concepções diferentes quanto às contra-indicações para analgesia em vítimas de trauma. O receio da analgesia mascarar um quadro clínico foi citado por $27,3 \%$ dos enfermeiros e por nenhum dos médicos entrevistados.

Observa-se na Tabela 5 que 88,6\% consideraram como principal parâmetro para avaliação da eficácia analgésica o "relato de dor do paciente". 
Ana Maria Calil Cibele A. de M. Pimenta

Tabela 5 - Distribuição da opinião dos profissionais sobre os principais parâmetros utilizados para a avaliação da eficácia analgésica em pacientes de trauma. (São Paulo, 2002)

\begin{tabular}{|c|c|c|c|c|c|c|}
\hline \multirow{2}{*}{ Parâmetros } & \multicolumn{2}{|c|}{ Enfermeiros } & \multicolumn{2}{|c|}{ Médicos } & \multicolumn{2}{|c|}{$\begin{array}{c}\text { Todos } \\
\text { Profissionais }\end{array}$} \\
\hline & $\mathbf{n}=\mathbf{2 2}$ & $\%$ & $\mathrm{n}=\mathbf{2 2}$ & $\%$ & $\mathrm{n}=44$ & $\%$ \\
\hline Relato de dor do paciente & 18 & 81,8 & 21 & 95,5 & 39 & 88,6 \\
\hline Agitação & 4 & 18,2 & 5 & 22,7 & 9 & 20,5 \\
\hline Características do fármaco & 5 & 22,7 & 2 & 9,1 & 7 & 15,9 \\
\hline Fáscies de dor & 4 & 18,2 & 2 & 9,1 & 6 & 13,6 \\
\hline Exame físico & 3 & 13,6 & 3 & 13,6 & 6 & 13,6 \\
\hline Sinais vitais & 2 & 9,1 & 3 & 13,6 & 5 & 11,4 \\
\hline Conforto & 4 & 18,2 & 2 & 9,1 & 4 & 9,1 \\
\hline Avaliação/reavaliação & 3 & 13,6 & 1 & 4,5 & 4 & 9,1 \\
\hline Escalas de avaliação & 2 & 9,1 & 1 & 4,5 & 3 & 6,8 \\
\hline Tranqüilidade & 2 & 9,1 & - & - & 2 & 4,5 \\
\hline Nível de consciência & - & - & 2 & 9,1 & 2 & 4,5 \\
\hline Presença de dor à manipulação & 1 & 4,5 & - & - & 1 & 2,3 \\
\hline
\end{tabular}

Comparando-se as respostas mais freqüentes "relato de dor do paciente", "agitação", "características do fármaco", "fáscies de dor" e "exame físico" (Tabela 6), notouse, não haver diferença significativa entre os profissionais.

Tabela 6 - Síntese das comparações das cinco respostas mais freqüentes apresentadas pelos profissionais na Tabela 5. (São Paulo, 2002)

\begin{tabular}{lc}
\hline Respostas & P* \\
\hline Relato de dor do paciente & 0,345 \\
Agitação & 1,000 \\
Características do Fármaco & 0,734 \\
Fáscies de dor & 0,664 \\
Exame físico & 1,000 \\
\hline
\end{tabular}

* Teste Qui-quadrado

Quanto aos principais parâmetros utilizados para avaliar a eficácia da analgesia, observouse semelhança entre as respostas dadas pelos profissionais, e estas são concordantes com a literatura, na maioria das citações. Entre as cinco respostas mais freqüentes, destaca-se a opinião dos médicos $(95,5 \%)$ e enfermeiros $(81,8 \%)$ de que o relato do paciente é o primeiro parâmetro de avaliação.

É importante que esse dado tenha sido identificado, uma vez que, sendo a dor um fenômeno subjetivo, a única forma eficaz de avaliação é através do relato do doente, por manifestação verbal ou outras formas. Da análise das respostas dos enfermeiros em relação à questão "indicações para o uso de analgesia" (Tabela 1) e à questão "os principais parâmetros utilizados para a avaliação da eficácia analgésica" (Tabela 5), notou-se coerência entre as respostas dos enfermeiros, isto é, indicação do uso de analgesia na vigência de dor e a opinião do paciente como principal parâmetro de avaliação da eficácia analgésica. Tal coerência não foi observada em relação aos médicos, pois estes só referiram ser a presença de dor critério para o uso de analgésicos em $9,1 \%$ das vezes.

A inexistência de um protocolo para a avaliação objetiva da dor aguda, no setor de emergência, constitui um problema para a estimação da eficácia analgésica e divergência entre os profissionais. É desejado que, num futuro próximo, tal implementação seja proposta.

Verifica-se na Tabela 7 que ambas categorias profissionais conferiram média $=4,00$ e mediana $=4,00$ para a ênfase dada à analgesia no trauma. Por meio do teste não-paramétrico de MannWhitney, observou-se que não houve diferença significativa entre os profissionais $(\mathrm{p}=0,1936)$. 
Tabela 7 - Distribuição da opinião dos profissionais quanto à ênfase conferida à analgesia em pacientes de trauma, numa escala de 0 a 10. (São Paulo, 2002)

\begin{tabular}{lccccc}
\hline \multicolumn{1}{c}{ Profissional } & Média & Desvio Padrão & Mediana & Mínimo & Máximo \\
\hline Médico & 4,00 & 3,13 & 4,00 & 0 & 10 \\
Enfermeiro & 4,64 & 2,06 & 4,00 & 0 & 10 \\
\hline
\end{tabular}

Observa-se na Tabela 8 que a média $=8,36 \mathrm{e}$ mediana $=8,00$ conferida pelos médicos foi menor que a pontuada pelos enfermeiros, respectivamen- te 9,18 e 9,00. O teste não-paramétrico de MannWhitney mostrou diferença significativa entre os profissionais $(\mathrm{p}=0,0496)$.

Tabela 8 - Distribuição da opinião dos profissionais em relação à ênfase que deveria ser conferida à analgesia em traumatologia, numa escala de 0 a 10. (São Paulo, 2002)

\begin{tabular}{lccccc}
\hline \multicolumn{1}{c}{ Profissional } & Média & Desvio Padrão & Mediana & Mínimo & Máximo \\
\hline Médico & 8,36 & 2,46 & 8,00 & 0 & 10 \\
Enfermeiro & 9,18 & 2,94 & 9,00 & 0 & 10
\end{tabular}

A análise das duas últimas questões reflete dois aspectos importantes e distintos da problemática da dor no trauma. A primeira refere-se à desvalorização conferida à analgesia em traumatologia, assinalada igualmente por médicos e enfermeiros, e a segunda, à importância de que a mesma deveria ter na prática profissional.

A maior parte dos profissionais considerou que o alívio da dor tem pouca ênfase em traumatologia, mas que a mesma deveria ser mais valorizada. Acredita-se que o pouco valor conferido a analgesia na dor aguda de um modo geral, e ao trauma de forma mais contundente, tenha sido amplamente discutido até o momento e confirmado em nosso meio pelos achados deste estudo.

Durante a aplicação do questionário, foi possível identificar, por parte dos profissionais entrevistados, um certo constrangimento em assumir a pouca importância conferida à dor no trauma. Tal constatação foi evidente em ambas as categorias profissionais e, de forma geral, os profissionais se mostraram interessados e abertos a mudanças de postura frente à negligência atual ao paciente com dor.

A solicitação de analgésicos para pacientes com dor, segundo os depoimentos dos enfermeiros, é feita aos médicos, em grande parte das vezes, após o atendimento inicial para que a mesma não seja "esquecida" durante o tratamento. Tal situação foi descrita como indesejada e muitas vezes constrangedora. Após revisão de literatura, constatou-se que essa situação não é um problema isolado e restrito à nossa realidade, mas sim, um entrave global vivenciado por profissionais em países desenvolvidos ${ }^{(15,24-25)}$. Tentativas isoladas de mudança de postura profissional frente à dor no trauma foram observadas, com sucesso, mas parecem pouco se comparadas à grande maioria dos serviços que permanecem sem um programa objetivo, efetivo e contínuo de avaliação e tratamento da dor aguda.

O ensino sobre dor e analgesia nas instituições de ensino de enfermagem se faz de forma inconstante e reduzida, fazendo com que os profissionais não assimilem essa informação como um valor e um parâmetro a ser incluído na sua prática profissional $^{(26)}$. A despeito das limitações e dificuldades da implementação de um programa institucional de controle de dor e analgesia, acredita-se na importância de sua concretização, sobretudo nas instituições de ensino, visando à melhoria da assistência e da formação de profissionais.

\section{CONCLUSÃO}

O estudo permitiu conhecer os conceitos de médicos e enfermeiros de um serviço de emergência sobre indicação e contra-indicação da analgesia no trauma, critérios para se avaliar a eficácia analgésica, e ênfase dada à analgesia no trauma. Existem divergências conceituais entre médicos e enfermeiros no que se refere às indicações analgésicas e concordância quanto as contra indicações e ao principal parâmetro para a avaliação da eficácia analgésica. Para os enfermeiros, "a presença de dor” é a principal razão de indicação analgésica, e para os médicos, "todas as situações, após a avaliação primária". As contra-indicações "nenhuma situação", "escore na Escala de Coma de Glasgow
Conceitos de enfermeiros e médicos de um serviço de emergência sobre dor e analgesia no trauma 
Ana Maria Calil

Cibele A. de M. Pimenta
< 9" e "trauma crânioencefálico" foram citadas como importantes para ambas as categorias profissionais. Os enfermeiros valorizaram a possibilidade da "analgesia mascarar o quadro" clínico. "O relato de dor do paciente" foi o principal parâmetro de avaliação da eficácia analgésica para ambas as categorias profissionais. Pouca ênfase

\section{REFERÊNCIAS}

(1) Clarke WC. A mensuração da dor. In: Kanner R. Segredos em clínica de dor. Porto Alegre: Artes Médicas; 1998. p. 35-50.

(2) McCaffery M, Pasero C. Appropriate pain control. [letter]. Am J Nurs 2001; 101(11):13.

(3) Kelly AM. A process approach to improving pain management in the emergency department: development and evaluation. J Accid Emerg Med 2001; 18(4):321-2.

(4) International Association for Study of Pain (IASP). Concensus development conference statement: the integrated approach to the management of pain. 1994; 6(3): (Document number - 491-292).

(5) Ready LB, Edwards WT. Dor no trauma. In: Ready LB. Tratamento da dor aguda. Rio de Janeiro: Revinter; 1995. Cap. 4, p. 27-30

(6) Teixeira MJ. Fisiopatologia da dor. Rev Med (São Paulo) 1995; 73(2):55-64

(7) Kanner R. Segredos em clínica de dor. Porto Alegre: Artes Médicas; 1998. Anamnese do paciente com dor; cap. 2, p. 31-4.

(8) Bonnet FA. A Dor no meio cirúrgico. Porto Alegre: Artes Médicas; 1993. Analgesia em traumatologia; cap. 13 , p. 187-92.

(9) Organização Mundial da Saúde (OMS). Manual de classificação estatística internacional de doenças e problemas de doenças relacionados à saúde. $10^{\mathrm{a}}$ ed. São Paulo: Centro Colaborador da OMS para Classificação de Doenças em Português; 1998. 2 v.

(10) Lebrão ML, Mello-Jorge MHP, Laurenti R. Acidentes e violência no Brasil. Rev Saúde Publica 1997; 31(4 Supl):26-37.

(11) Andrade SM. Acidentes de transporte em Londrina, Paraná: análise das vítimas dos acidentes e das fontes de informação. [tese]. São Paulo (SP): Faculdade de Saúde Pública da USP; 1998.

(12) São Paulo (Cidade) Prefeitura do Município de São Paulo. Programa de Aprimoramento das Informações de Mortalidade do Município de São Paulo (PRO-AIM). Alguns aspectos da mortalidade no Município de São Paulo em 1998. São Paulo; 1999.

(13) Fundação IBGE. Anuário estatístico do Brasil. Rio de Janeiro; 2000. v.59. à analgesia no trauma foi observada. No entanto, médicos e enfermeiros consideram que maior atenção deve ser dada à questão. Considera-se fundamental a valorização da dor pelos profissionais que atuam no setor de emergência, contribuindo para a qualidade do atendimento nesse setor.

(14) Pimenta CAM, Carvalho MB, Fogaça V. Dor e analgesia no atendimento pré-hospitalar às vítimas de acidente de causa externa. Âmbito Hosp 2003; 14(160):8-13.

(15) McCaffery M, Pasero C. How to choose the best route for an opioid. Nursing 2000; 12(30):34-9.

(16) Teixeira MJ, Fonoff E, Erich T, Lepski G, Marcon RM, Rocha RO. Dor no atendimento em prontosocorro. Avaliação do conhecimento da equipe de saúde sobre dor, analgesia e procedimentos prescritos para o controle. Rev Med 1999; 78(3):359-63.

(17) Horta WA. Humanização. [editorial]. Enferm Novas Dimens 1977; 3(4):III

(18) Pasero C, McCaffery M, Gordon DB. Build Institutional Commitment to Improving Pain Management. Nurs Manage 1999; 30(1):27-33.

(19) Ward KR, Yealy DM. Systemic analgesia and sedation in managing orthopedic emergencies. Emerg Med Clin North Am 2000; 18(1):141-65.

(20) Alpen MA, Morse C. Managing the pain of traumatic injury. Crit Care Nurs Clin North Am 2001; 3(2):243-57.

(21) Boutros F, Redelmeier DA. Effects of trauma cases on the care of patients who have chest pain in an emergency department. J Trauma 2000; 48(4):649-53.

(22) Espinosa V, Ortega F, Ávila E, Naranjo R. Traumatismos por accidentes de transito: revision retrospectiva de 550 casos. Medicina 1996; 2(3):152-7.

(23) Carvalho WB, Troster EJ. Sedação e analgesia no pronto-socorro. J Pediatr 1999; 75(Supl 2): 295-306.

(24) Pasero C, McCaffery M. Multimodal balanced analgesia in the critically ill. Crit Care Nurs Clin North Am 2001; 13(2):195-206.

(25) Slaughter A, Pasero C, Montgomery R. Unacceptable pain levels: approaches to prompting pain relief. Am J Nurs 2002; 102(5):75-7.

(26) Bernardo CLE. Dor: o ensino do tema em cursos de graduação em enfermagem da Região Sudeste do Brasil. [dissertação]. São Paulo (SP): Escola Paulista de Medicina UNIFESP; 1998. 